Trauma Berufskrankh $2007 \cdot 9$ [Suppl 3]:

S264-S273

DOI 10.1007/s10039-007-1280-x

Online publiziert: 6. September 2007

(c) Springer Medizin Verlag 2007
H. Bauer

Deutsche Gesellschaft für Chirurgie, Berlin

\section{Chirurgie in neuen Strukturen}

\section{Auswirkungen auf den Nachwuchs}

Zum Thema Chirurgie in neuen Strukturen und deren Auswirkungen auf den Nachwuchs sollen 2 Schwerpunkte diskutiert werden:

- die Chirurgie als neu strukturiertes Gebiet im Rahmen der eben erst umgesetzten Weiterbildungsordnung und

- die Herausforderungen, die neue Versorgungsstrukturen an die Chirurgie und die Chirurgen stellen.

\section{Struktur des Gebiets Chirurgie in der neuen Muster- weiterbildungsordnung}

\section{Entwicklung seit 1969}

Der Lebenszyklus einer von der Bundesärztekammer verabschiedeten und von den einzelnen Landesärztekammern umzusetzenden Musterweiterbildungsordnung (MWBO) betrug seit Änderung der Bundesärzteordnung 1969 mit Einführung von ersten Teilgebieten in der Chirurgie (Kinder- und Unfallchirurgie) durchschnittlich 10 Jahre. 1978 wurden die weiteren Teilgebiete Gefäßchirurgie, Thoraxchirurgie und Kardiovaskularchirurgie definiert, 1988 kam das Teilgebiet Plastische Chirurgie dazu. Bei weitergehender Spezialisierung der chirurgischen Disziplinen, getragen von ihren eigenen wissenschaftlichen Fachgesellschaften, erfolgte bereits nach 5 Jahren, 1993, die Festlegung der eigenständigen Fachgebiete Kinder-, Herz- und Plastische Chirurgie mit einer Schwerpunktlösung im $\mathrm{Ge}$ biet Chirurgie für die Gefäß-, Thorax-
, Unfall- und Viszeralchirurgie. Der Zugang zum Schwerpunkt Thoraxchirurgie war sowohl über das Gebiet Chirurgie als auch über das der Herzchirurgie möglich. Nach weiteren 10 Jahren erfolgte mit der MWBO 2003 eine grundlegende Neugliederung des Gebiets Chirurgie, deren Umsetzung in allen Landesärztekammern immerhin bis 2006 dauerte (• Abb. 1).

\section{8-Säulen-Modell}

Die Definition der Gebietsgrenzen hat in der MWBO 2003 eine wesentliche strukturelle Neuerung erfahren. Innerhalb eines Gebiets können verschiedene Kompetenzen erworben werden. Im Gebiet Chirurgie zählen dazu nur die verschiedenen Facharzt- und Zusatzweiterbildungen, eine Schwertpunktweiterbildung wie in anderen Gebieten (z. B. Innere Medizin oder Frauenheilkunde und Geburtshilfe) gibt es hier nicht. Die vorher eigenständigen Gebiete Kinder-, Herz- und Plastische Chirurgie sowie die Orthopädie in ihrer neuen gemeinsamen Struktur mit der Unfallchirurgie haben sich unter den Bedingungen einer 2-jährigen Basischirurgie („,common trunk“), für alle verpflichtend und mit den gleichen $\mathrm{zu}$ vermittelnden Inhalten, bereit gefunden, als gleichberechtigte Partner in das neu definierte „Haus“ der Chirurgie einzuziehen (8-Säulen-Modell). Der „Charme“ dieses Konzepts liegt auch darin, dass es für weitere operative Disziplinen offen ist (- Abb. 2).

Eine Befugnis zur Weiterbildung in der Basischirurgie ist grundsätzlich in al- len Facharztsäulen der Chirurgie möglich, sofern die dort vorgeschriebenen Inhalte vermittelt werden können. Für die halbjährigen Weiterbildungszeiten Intensivmedizin und Notfallaufnahme kann dies allerdings häufig nur über Verbundermächtigungen gemeinsam mit anderen chirurgischen Fachdisziplinen oder der Anästhesie sichergestellt werden.

\section{Facharztausbildung}

Die spezifische Facharztkompetenz wird in einem auf dem "common trunk“ aufbauenden 4-jährigen „special trunk“ erworben, in dem auch 1-jährige Anerkennungen in einem "Gegenfach" möglich sind. Innerhalb dieser vorgegebenen Mindestweiterbildungszeiten können infolge der zunehmenden Spezialisierung allerdings nicht mehr alle Inhalte vermittelt werden. Bestimmte hoch spezialisierte Leistungen sind somit nicht zwingend Gegenstand der obligatorischen Weiterbildung zum Erwerb einer Facharztkompetenz in einer der Säulen. Besondere Kenntnisse und Erfahrungen müssen hier im Rahmen von Zusatzweiterbildungen erworben werden (z. B. Spezielle Unfallchirurgie, Spezielle Orthopädische Chirurgie).

Die chirurgischen Facharztspezialitäten haben in ihrer Gesamtheit Zugang zu insgesamt 26 Zusatzweiterbildungen, deren Erwerb v. a. im Rahmen einer Niederlassung attraktiv ist (• Tab. 1). 
Tab. 1 Mögliche Zusatzweiterbil-

dungen für die verschiedenen Säulen des

Gebiets Chirurgie

\begin{tabular}{l} 
Akupunktur \\
Hämostaseologie \\
Handchirurgie \\
\hline Homöopathie \\
Infektiologie \\
\hline Intensivmedizin \\
\hline Kinderorthopädie \\
\hline Labordiagnostik \\
\hline MRT \\
\hline Manuelle Medizin/Chirotherapie \\
\hline Medikamentöse Tumortherapie \\
\hline Medizinische Informatik \\
\hline Naturheilverfahren \\
\hline Notfallmedizin \\
\hline Orthopädische Rheumatologie \\
\hline Palliativmedizin \\
\hline Phlebologie \\
\hline Physikalische Therapie und Balneologie \\
\hline Proktologie \\
\hline Rehabilitationswesen \\
\hline Röntgendiagnostik \\
\hline Sozialmedizin \\
\hline Spezielle Orthopädische Chirurgie \\
\hline Spezielle Schmerztherapie \\
\hline Spezielle Unfallchirurgie \\
\hline Sportmedizin \\
\hline Plastische Operationen: für HNO oder MKG \\
(Anträge von Dermatologie und Gynäkologie \\
abgelehnt) \\
\hline
\end{tabular}

\section{Fachgesellschaft}

Ausgehend vom 8-Säulen-Modell des Gebiets Chirurgie in der MWBO 2003 ist es gelungen, auch die diese Facharztsäulen tragenden eigenständigen wissenschaftlichen Gesellschaften in der Deutschen Gesellschaft für Chirurgie (DGCH) zu vereinen. Neben der Funktion als Dachgesellschaft ist der DGCH noch die Aufgabe verblieben, die Allgemeine Chirurgie als wissenschaftliche Gesellschaft zu vertreten, nachdem diese als einzige Facharztsäule von keiner eigenständigen Fachgesellschaft repräsentiert wird.

\section{Säule Allgemeine Chirurgie}

\section{Als Kompromisslösung}

Im Gebiet Chirurgie stellt die Säule Allgemeine Chirurgie eine unter den chirurgischen Fachgesellschaften abgestimm-

Trauma Berufskrankh 2007 · 9[Suppl 3]: S264-S273 DOI 10.1007/s10039-007-1280-x

(c) Springer Medizin Verlag 2007

\section{H. Bauer \\ Chirurgie in neuen Strukturen. Auswirkungen auf den Nachwuchs}

\section{Zusammenfassung}

Zum Thema Chirurgie in neuen Strukturen und deren Auswirkungen auf den Nachwuchs werden 2 Schwerpunkte diskutiert, die Chirurgie als neu strukturiertes Gebiet im Rahmen der neuesten Weiterbildungsordnung und die Herausforderungen, die neue Versorgungsstrukturen an die Chirurgie und die Chirurgen stellen. Das Gebiet der Chirurgie hat sich seit 1969 durch Einführung immer neuer Musterweiterbildungsverordnungen entscheidend zum heutigen 8-Säulen-Modell gewandelt. Die Säule „Allgemeine Chirurgie" stellt in diesem jetzt gültigen Modell eine Kompromisslösung der verschiedenen Fachgesellschaften dar. Ihre Perspektiven erscheinen momentan eher ungewiss, hier sind eine Neudefinition des Aufgabenspektrums sowie entsprechende Anpassungen der MWBO erforderlich. Die Qualität und Effizienz der chirurgischen Weiterbildung müssen verbessert werden. Zur Effizienzsteigerung müssen Aufgaben delegiert und neue nichtärztliche Berufsbilder internalisiert werden. Die neuen Versorgungsformen erfordern ein Umdenken und werden zu neuen Spezialisierungsrichtungen, Fallzahlkonzentration und Zentrumsbildung führen. Letztendlich ist eine professionelle Neuorientierung unumgänglich.

\section{Schlüsselwörter}

Weiterbildungsordnung $\cdot$ Versorgungsstrukturen · 8-Säulen-Modell • „Allgemeine Chirurgie" · Professionelle Neuorientierung

\section{Surgery with the new structures. Implications for new surgeons}

\section{Abstract}

The topic of surgery in new structures and their implications for surgeons in training is discussed with two main emphases: surgery as a newly structured sector in the context of the latest regulations on continuing education and the challenges that surgery and surgeons are confronted with by the new structures in care. The surgery sector has changed decisively since 1969 , owing to the introduction of more and more regulations on continuing education, culminating in the present 8-column model. The General Surgery column in this present model is a compromise devised by the various specialist societies. Its perspectives seem rather uncertain at the moment, and a fresh definition of the range of its tasks and appropriate adaptations of the model continuing education regulations are needed. The quality and efficiency of continuing education in surgery must be improved. To enhance efficiency tasks must be delegated and descriptions of new, nonmedical occupations must be integrated. The new forms of care require a rethink and will lead to new specialisms, and to the concentration of case numbers and formation of centres. UItimately, reorientation of all professions involved will be inevitable.

\section{Keywords}

Regulations on continuing education . Structures of care $\cdot 8$-Column model $\cdot$ General surgery $\cdot$ New professional orientation 
Neuer Facharzt

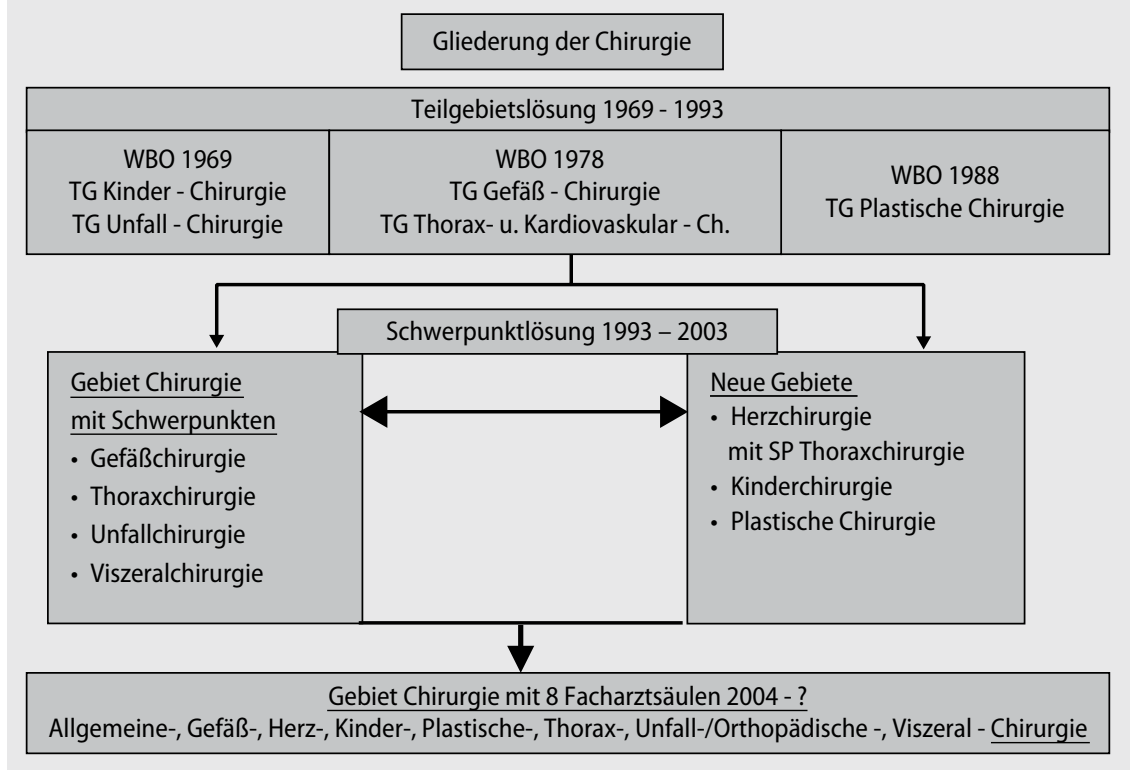

Abb. 1 \ Verlauf der Aufgliederung der Chirurgie durch MWBO seit 1969

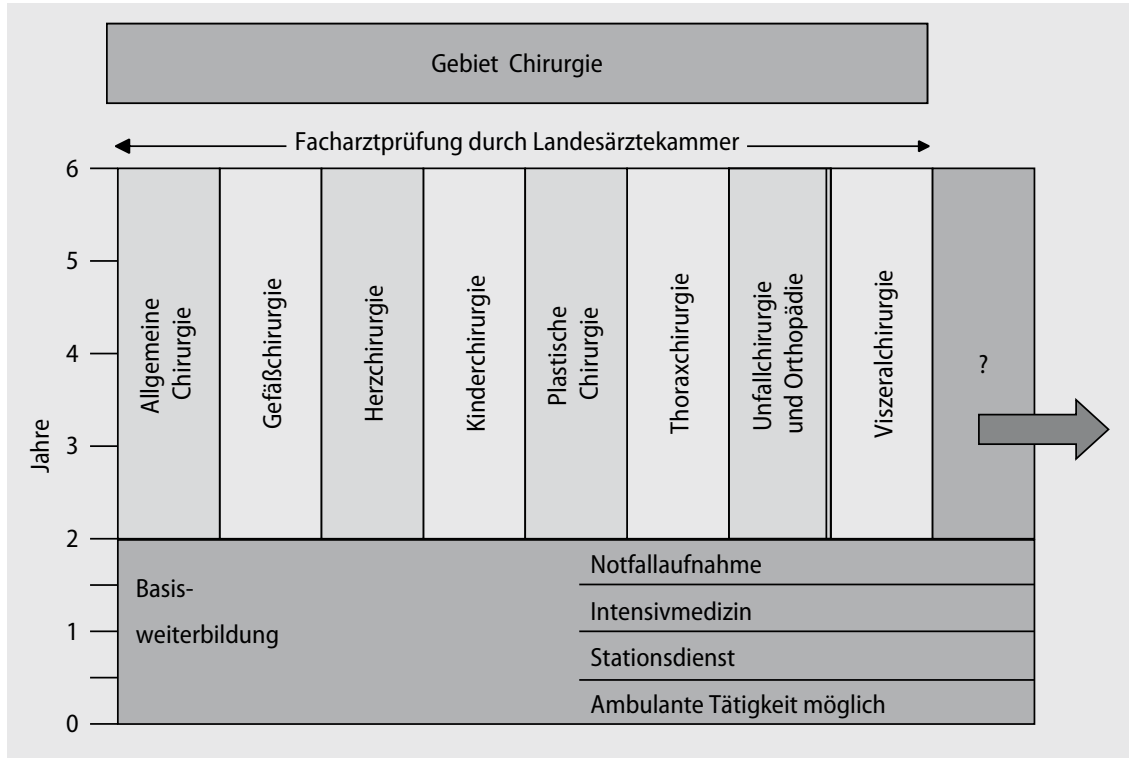

Abb. 2 \ 8-Säulen-Modell der Chirurgie

te Kompromisslösung dar. Ausdruck dafür sind schon die semantischen Bemühungen, nicht mehr von „Allgemeinchirurgie" und "Allgemeinchirurg" zu sprechen, ein Begriff, der dem chirurgischen Alleskönner alter Prägung mit einem entsprechenden umfassenden Kompetenzanspruch zugeordnet wurde. Der Begriff „Allgemeine Chirurgie“ wiederum war früher belegt für die Grundlagen der Chirurgie, wie sie in jedem Lehrbuch der speziellen Chirurgie vorangestellt wurden. Diese Inhalte sind heute der Basischirurgie (,common trunk“) und damit allen 8 Facharztsäulen zugeordnet.
Der Kompromiss der Säule Allgemeine Chirurgie war z. T. auch europäischen Regelungen geschuldet, um die Neugliederung des Fachgebiets in Europa ratifizierungsfähig zu machen. Allgemeinchirurgie („general surgery“) stellt in den meisten Ländern die chirurgische Versorgung der Bevölkerung sicher. Als chirurgische Disziplin ist sie durch die EU-Direktive 93/16 und Folgeregelungen mit $\mathrm{Si}$ chergestellung einer gegenseitigen Anerkennung innerhalb der EU-Länder definiert. Die Bedeutung der EU-Kompatibilität wird auch in dem jahrelangen Streit um einen Facharzt für Innere Medizin als eigenständigem Weiterbildungsgang neben den verbindlich zu erwerbenden internistischen Schwerpunkten deutlich. Nachdem dieser Facharzt in der neuen MWBO nicht mehr vorgesehen war, wurde nach heftigem Widerstand der Fachgesellschaft der Allgemeininternist auf dem Deutschen Ärztetag 2007 wieder eingeführt.

Eine Kompromisslösung waren aber auch die Festlegungen zur Allgemeinen Chirurgie im Paragraphenteil der MWBO sowie die dazu in den Richtlinien festgeschriebenen Inhalte. In $\$ 6.1$ ist vorgesehen, dass nach Ableistung der 2 Jahre Basischirurgie von den verbleibenden 4 Jahren Mindestweiterbildungszeit in der Allgemeinen Chirurgie je 12 Monate in dafür befugten Abteilungen für Viszeralchirurgie und Unfallchirurgie/Orthopädie sowie weitere 24 Monate in diesen oder anderen Facharztweiterbildungen des Gebiets Chirurgie abgeleistet werden müssen. Ob dabei bei wörtlicher Interpretation der MWBO auch die Allgemeine Chirurgie einzubeziehen ist, war bis jetzt strittig. Nach Auffassung der Weiterbildungsgremien der Bundesärztekammer schließt das Wort ",andere“ in diesem Zusammenhang eindeutig die Facharztweiterbildung in der Allgemeinen Chirurgie aus. In einigen Landesärztekammern, denen die vom Deutschen Ärztetag beschlossene MWBO nur als Richtschnur dient, wird dies anders gesehen. Länderspezifisch kann es also neben der pflichtgemäßen 1-jährigen Rotation in die Viszeral- und Unfallchirurgie/Orthopädie eine bis zu 2-jährige anerkannte Weiterbildung in der Allgemeinen Chirurgie geben.

Eine verbindliche Festlegung hierzu existierte bisher nicht. Die Allgemeine Chirurgie wäre damit die einzige Facharztsäule, in der im Rahmen der 4 Jahre „special trunk“ Allgemeine Chirurgie Ärzte/-innen dieser Fachrichtung nicht eigenständig weitergebildet werden könnten, auch wenn eine Vermittlung der erforderlichen Inhalte gewährleistet wäre. Aufgrund dieser Situation wären v. a. die an Krankenhäusern der Grund- und Regelversorgung noch in überwiegender Anzahl existierenden ungeteilten chirurgischen Abteilungen von der Weiterbildung in der Allgemeinen Chirurgie ausgeschlossen. Gerade die Einrichtungen, 
die in den Flächenstaaten einen Großteil der chirurgischen Versorgung zu tragen haben und für die auch künftig zur Sicherstellung eines wohnortnahen Angebots noch Bedarf besteht, würden mangels anerkannter Weiterbildungsmöglichkeiten jegliche Attraktivität für den chirurgischen Nachwuchs verlieren.

Auf Intervention unserer gemeinsamen Weiterbildungskommission von Fachgesellschaften und Berufsverband wurde nun Klarheit geschaffen. Im Sinne eines Interpretationsbeschlusses wurde eine von der Ständigen Konferenz Ärztliche Weiterbildung der Bundesärztekammer (StäKo) vorgeschlagenen Formulierung beschlossen, die neben den pflichtgemäßen 1-jährigen Rotationen in die Viszeral- und Unfallchirurgie auch die Möglichkeit einer 2-jährige Weiterbildungszeit in der Allgemeinchirurgie innerhalb der 48 Monate „special trunk“ Allgemeine Chirurgie eröffnet [3].

\section{Perspektiven}

Diese formalen weiterbildungsrelevanten Probleme der Allgemeinen Chirurgie in der neuen MWBO sowie die noch zu diskutierenden Inhalte mit ihren Überschneidungen mit den anderen Facharztsäulen insbesondere in der „Chirurgie des Häufigen “ sind die eine Seite. Die entscheidende Frage ist, welche beruflichen Perspektiven haben heute überhaupt noch angehende Chirurgen/-innen, die sich meist schon während ihrer Weiterbildungszeit in der Basischirurgie anstelle einer frühzeitigen Spezialisierung in einer der anderen Säulen für die Allgemeine Chirurgie entscheiden möchten bzw. wo wird der Allgemeinchirurg mit dieser Qualifikation heute noch gebraucht? Hat er eine Zukunft in einer immer mehr von Spezialisierung, Zentralisierung, Interdisziplinarität, Sektor übergreifenden Integrationsmodellen und Fallzahlkonzentration geprägten Krankenversorgung? Selbst Abteilungen in Krankenhäusern der Grundversorgung werden heute, wenn schon nicht in eigenständige Untereinheiten geteilt, zunehmend paritätisch von Unfallchirurgen und Viszeralchirurgen geleitet. Fachübergreifende Bereitschaftsdienste auf Oberarztebene sind dabei noch die Regel, was deshalb noch funktioniert, weil diese Fachärzte, ob nun
Viszeral- oder Unfallchirurg, nach der bisherigen Schwerpunktlösung immer noch auch Fachärzte für Chirurgie alter Prägung sind.

Noch gehören nach einer aktuellen Statistik der Bundesärztekammer die Fachärzte für Allgemeinchirurgie/Allgemeine Chirurgie neben den neuerdings gemeinsam aufgeführten Unfallchirurgen und Orthopäden zahlenmäßig zur stärksten Gruppe im Gebiet Chirurgie (• Tab. 2). Neben den klinischen Möglichkeiten für Generalisten im Sinne des jetzigen Facharztes für Allgemeine Chirurgie besteht ein Bedarf im ambulanten Bereich am ehesten als chirurgischer Partner in medizinischen Versorgungszentren, während die Niederlassung in eigener Praxis aufgrund der nicht erreichbaren D-ArztZulassung bzw. der Konkurrenz von Spezialisten aus anderen chirurgischen Fächern, ganz abgesehen von den ökonomischen Rahmenbedingungen, unattraktiv bleiben dürfte. Alternative Tätigkeitsfelder für Allgemeine Chirurgen bei Krankenkassen und Versicherungen, bei Behörden, in der Industrie, bei Beratungsunternehmen oder in der Krankenhausverwaltung entsprechen sicher nicht dem primären Berufswunsch, sondern sind eher Sekundärentscheidungen im Berufsleben. Ein echter Bedarf an breit ausgebildeten Allgemeinchirurgen besteht, ähnlich wie in Entwicklungsländern, sicher bei der Bundeswehr im Rahmen ihrer zunehmenden Einsatzaufgaben, wobei auch hier noch intensiv diskutiert wird, inwieweit Spezialisten zur Gewährleistung einer dem Zivilbereich vergleichbaren Versorgungsqualität unverzichtbar sind.

\section{Als Chirurgie des Häufigen}

Ganz entscheidend ist die Frage, wie die Allgemeine Chirurgie mit den ihr in der jetzigen MWBO zugewiesenen Inhalten Bestand haben soll. Aus allen Facharztsäulen finden sich Inhalte, die im Richtlinienkatalog auch der Allgemeinen Chirurgie zugeordnet sind. Das gilt zum weitaus überwiegenden Teil für die Viszeralchirurgie und die Unfallchirurgie/Orthopädie. Soll die Allgemeine Chirurgie als chirurgisches Querschnittsfach eine $\mathrm{Zu}$ kunft haben, muss sie, orientiert auch an internationalen Curricula, unter Berücksichtigung der angesprochenen, mit den
Tab. 2 Anteil der einzelnen Fachrich-

tungen im Gebiet Chirurgie (Auszug

Ärztestatistik der BÄK 2006)

\begin{tabular}{ll}
\hline $\begin{array}{l}\text { Fachrichtung } \\
\text { Chirurgie/Allgemeine } \\
\text { Chirurgie }\end{array}$ & $\begin{array}{l}\text { Anzahl der Ärzte } \\
11.419\end{array}$ \\
\hline $\begin{array}{l}\text { Gefäßchirurgie } \\
\begin{array}{l}\text { Herzchirurgie/Kardio- } \\
\text { vaskularchirurgie }\end{array}\end{array}$ & 7041 \\
\hline $\begin{array}{l}\text { Kinderchirurgie } \\
\text { Orthopädie/Unfallchir- } \\
\text { urgie }\end{array}$ & 12.043 \\
\hline $\begin{array}{l}\text { Plastische und ästhe- } \\
\text { tische Chirurgie }\end{array}$ & 655 \\
\hline $\begin{array}{l}\text { Thoraxchirurgie } \\
\text { Viszeralchirurgie }\end{array}$ & 232 \\
\hline $\begin{array}{l}\text { Gesamtzahl der im } \\
\text { Gebiet Chirurgie berufs- } \\
\text { tätigen Ärzte }\end{array}$ & 28.414 \\
aOhne Spezialgebiet Rheumatologie 533 \\
\hline
\end{tabular}

Nachbarfächern konkurrierenden Schnittmengen ein anderes Profil gewinnen. Allgemeine Chirurgie oder Allgemeinchirurgie („general surgery“) ist auch nach EU-Verständnis nicht nur Basischirurgie, wie sie als „common trunk“ für die chirurgischen Facharztspezialitäten definiert wurde. Sie kann nicht allein darauf reduziert werden, auch nicht auf einen so genannten „enlarged common trunk“, ohne ihre Existenz bzw. eigenständige Berechtigung in Frage zu stellen [3].

In der Allgemeinchirurgie besteht, abhängig von nationalen (EU) und regionalen Gegebenheiten (Ballungszentren, Flächenländer), ein unterschiedlicher Leistungsbedarf. Zum typischem Eingriffsspektrum gehören bei uns derzeit:

- Hernien,

- Appendektomien,

- Cholezystektomien,

- Kolon- und Strumaresektionen sowie

- Notfalleingriffe beim akuten Abdomen (Hohlorganperforationen, Ileus, Blutungen),

- in der Traumaversorgung die Behandlung von Frakturen des Schenkelhalses und unkomplizierten Frakturen z. B. des Sprunggelenks oder großer Röhrenknochen.

Im Bereich der Gefäßchirurgie sollten Embolektomien vom Allgemeinchirurgen beherrscht werden. Damit bestehen in der "Chirurgie des Häufigen“ große Über- 


\begin{tabular}{|c|c|c|c|}
\hline Gebiet & Eingriffsart & & Anzahl \\
\hline \multirow{6}{*}{$\begin{array}{l}\text { Viszeral- } \\
\text { chirurgie }\end{array}$} & Kopf und Hals & & 25 \\
\hline & \multirow{5}{*}{$\begin{array}{l}\text { Bauchwand und Bauchhöhle } \\
\text { (einschließlich Resektionen, Übernähungen, Exstirpationen und Exzisionen mittels } \\
\text { konventioneller, endoskopischer und interventioneller Techniken, z. B. Lymphkno- } \\
\text { tenexstirpationen, Portimplantationen, Entfernung von Weichteilgeschwülsten, ex- } \\
\text { plorative Laparotomien, Magen-, Dünn- und Dickdarmresektionen, Notversorgung } \\
\text { von Leber- und Milzverletzungen, Appendektomien, Anus-praeter-Anlage, Hämor- } \\
\text { rhoidektomie, periproktitische Abszessspaltungen, Fistel- und Fissurversorgung) }\end{array}$} & Gesamt & 200 \\
\hline & & Davon: & \\
\hline & & Cholezystektomien & 25 \\
\hline & & Herniotomien & 30 \\
\hline & & $\begin{array}{l}\text { Mitwirkungen bei Eingriffen hö- } \\
\text { herer Schwierigkeitsgrade }\end{array}$ & \\
\hline \multirow{6}{*}{$\begin{array}{l}\text { Unfall- } \\
\text { chirurgie/ } \\
\text { Orthopädie }\end{array}$} & \multirow[t]{4}{*}{ Stütz- und Bewegungssystem } & Osteosynthesen & \multirow[t]{4}{*}{100} \\
\hline & & Metallentfernung & \\
\hline & & Exostosenabtragung & \\
\hline & & Amputationen & \\
\hline & \multicolumn{2}{|l|}{ Reposition von Frakturen und Luxationen } & 50 \\
\hline & \multicolumn{2}{|l|}{ Versorgung von großen Wunden } & 25 \\
\hline \multirow{2}{*}{$\begin{array}{l}\text { Gefäßchirurgie } \\
\text { (Gefäße und } \\
\text { Nerven) }\end{array}$} & \multicolumn{2}{|l|}{ Varizen } & 25 \\
\hline & \multicolumn{2}{|l|}{ Embolektomien } & \\
\hline \multirow[t]{2}{*}{ Thoraxchirurgie } & \multicolumn{2}{|l|}{ Brustwand einschließlich Thorakotomien und Thoraxdränagen } & 10 \\
\hline & \multicolumn{2}{|l|}{ Mitwirkung bei Eingriffen höherer Schweregrade } & 20 \\
\hline
\end{tabular}

schneidungen v. a. mit der Viszeral- und Unfallchirurgie, denn die genannten Eingriffe sind auch die häufigsten operativen Leistungen, die der sich spezialisierende Viszeral- oder Unfallchirurg während seiner Weiterbildungszeit zu erbringen hat (- Tab. 3). Diese Tatsache der sich überlappenden Leistungsspektren führt da$\mathrm{zu}$, dass für bestimmte operative Bereiche künftig unterschiedliche Qualifizierungswege bestehen werden. Dass daraus auch zwangsläufig eine unterschiedliche Versorgungsqualität resultieren wird, wird vermutet, ist allerdings (noch) nicht bewiesen. Ob im Rahmen der vorgeschriebenen Rotationen (abgesehen von den Schwierigkeiten, überhaupt genügend Weiterbildungsstellen für angehende Allgemeinchirurgen in den anderen Facharztspezialitäten bereitstellen zu können) auch ausreichende Kenntnisse und Erfahrungen für diese Teilbereiche der Allgemeinen Chirurgie erworben und so chirurgische Grund- und Breitenversorgung in der erforderlichen Qualität gewährleistet werden können, ist zu bezweifeln. Der operative Kenntnis- und Erfahrungsstand am Ende des Pflichtrotationsjahrs wird keineswegs dem Niveau entsprechen können, das als Basis für die viszeral- bzw. unfallchirurgische Qualifikation des Allgemeinchirurgen $\mathrm{zu}$ fordern ist [3].

Bei den Inhalten der Allgemeinen Chirurgie, wie sie jetzt in den Richtli- nien zur MWBO definiert sind, ergeben sich nicht nur Probleme für den chirurgischen Nachwuchs in der Weiterbildungszeit, sondern auch hinsichtlich einer künftigen flächendeckenden chirurgischen Versorgung und späterer Berufsperspektiven. Ein denkbarer Lösungsweg wäre eine doppelte Facharztqualifikation, wobei die Allgemeine Chirurgie entweder mit der Viszeralchirurgie oder mit der Unfallchirurgie/Orthopädie kombiniert werden kann. Bei der Möglichkeit, Teile der speziellen Weiterbildung in der Viszeral- bzw. Unfallchirurgie in der Weiterbildungszeit zum Allgemeinen Chirurgen neben der pflichtgemäßen Rotation ins Gegenfach zu „versenken“, wäre dieser doppelte Facharzt in 9 Jahren Mindestweiterbildungszeit zu erreichen (• Abb. 3). Die aufzuwendende Zeit entspräche damit der Gesamtweiterbildungszeit für einen Unfallchirurgen/Orthopäden, der die Zusatzweiterbildung Spezielle Unfallchirurgie oder Spezielle Orthopädische Chirurgie erwerben will, bei möglicher Ableistung von 12 Monaten der 3-jährigen $\mathrm{Zu}$ satzweiterbildung während der Facharztweiterbildung zum Unfallchirurgen/Orthopäden. Die Viszeralchirurgie hat hier insofern ebenfalls Handlungsbedarf, als spezielle Eingriffe höherer Schwierigkeitsgrade wie Ösophagus- oder Pankreasresektionen, aber auch spezielle chirurgische Onkologie oder endokrine Chirur- gie in 4 Jahren spezieller Weiterbildungszeit nicht ausreichend vermittelt werden können. Hier wäre eine Zusatzweiterbildung „Spezielle Viszeralchirurgie“, vergleichbar zur Unfallchirurgie/Orthopädie, ein Lösungsansatz.

Bei den derzeitigen chirurgischen Versorgungsstrukturen v. a. in den Krankenhäusern der Grund- und Regelversorgung außerhalb der Ballungsgebiete sind die für die Allgemeine Chirurgie definierten traumatologischen Inhalte noch unverzichtbar. In absehbarer Zeit werden sich aber unter der Prämisse einer sicherzustellenden flächendeckenden fachärztlichen Versorgungsqualität auch hier partnerschaftliche Leitungsstrukturen dieser Abteilungen durch einen viszeralchirurgisch geprägten Allgemeinchirurgen und einen Facharzt für Unfallchirurgie/Orthopädie durchsetzen. Solche „Doppelspitzen“ werden die Regel auch in der Breitenversorgung werden.

\section{Ausblick}

Die flächendeckende Einführung der neuen MWBO hat eben erst begonnen. Es gibt dabei genug Probleme, was etwa die Sicherstellung, Strukturierung und Vermittlung der Basischirurgie in den einzelnen Fächern oder Übergangsregelungen im neuen Fach Unfallchirurgie/Orthopädie anbelangt. Die bundeseinheitlichen Vorgaben der MWBO haben dabei in 
der föderalen Vielfalt der 17 Landesärztekammern z. T. deutlich unterschiedliche Umsetzungen erfahren. Für (voreilige) Schlüsse bezüglich der Überlebensfähigkeit der Allgemeinen Chirurgie und eine Bedarfsabschätzung für derart qualifizierte Chirurgen ist es sicher noch zu früh. Die Einsicht, dass die Bedingungen für die neu definierte Säule Allgemeine Chirurgie andere sind als für das frühere Gebiet Chirurgie, hat sich noch nicht genügend durchgesetzt. Soll sie langfristig eine $\mathrm{Zu}-$ kunft haben, wofür vieles nicht nur unter den Aspekten der Europakompatibilität spricht, müssen wir trotz längst nicht erreichter Halbwertszeit der üblichen Dauer einer neu definierten MWBO jetzt schon über deren nächste Anpassung bzw. Änderung nachdenken [1].

\section{Steigerung von Qualität und Effizienz der chirurgischen Weiterbildung}

Bundesweit wollen nur noch etwa $6 \%$ der jungen Ärzte die Laufbahn des Chirurgen einschlagen. Das Interesse der Studenten an der Chirurgie nimmt im Lauf des Studiums und v. a. nach den Erfahrungen im Praktischen Jahr wie auch in anderen westlichen Industrieländern kontinuierlich ab (• Abb. 4). Es ist absehbar, dass uns in Deutschland bereits in wenigen Jahren ein Chirurgenmangel bevorstehen wird, wenn es nicht gelingt, die Attraktivität des Fachs für Studienabgänger wieder zu steigern und v. a. auch mehr Frauen für die Chirurgie zu interessieren [4].

Für diese mangelnde Attraktivität des Fachs ist zum einen die hohe Belastung der Chirurgen verantwortlich, die von den Studenten als deutlich höher als in anderen Fächern eingeschätzt wird. Der chirurgische Alltag ist geprägt von ständig zunehmender Arbeitsverdichtung. Moderne Operationsverfahren und neue Therapiekonzepte, v. a. aber der ökonomische Druck mit immer strafferen Ablauforganisationen und Verweildauerverkürzungen führen nicht nur zu einem gesteigerten „Patientenumsatz “ und höherer „Produktivität“ im Krankenhaus. Ein Großteil des neben der operativen Tätigkeit verbleibenden Arbeitstags ist mit Dokumentation und zermürbender Bürokratie wie etwa der zunehmenden Anfragen-

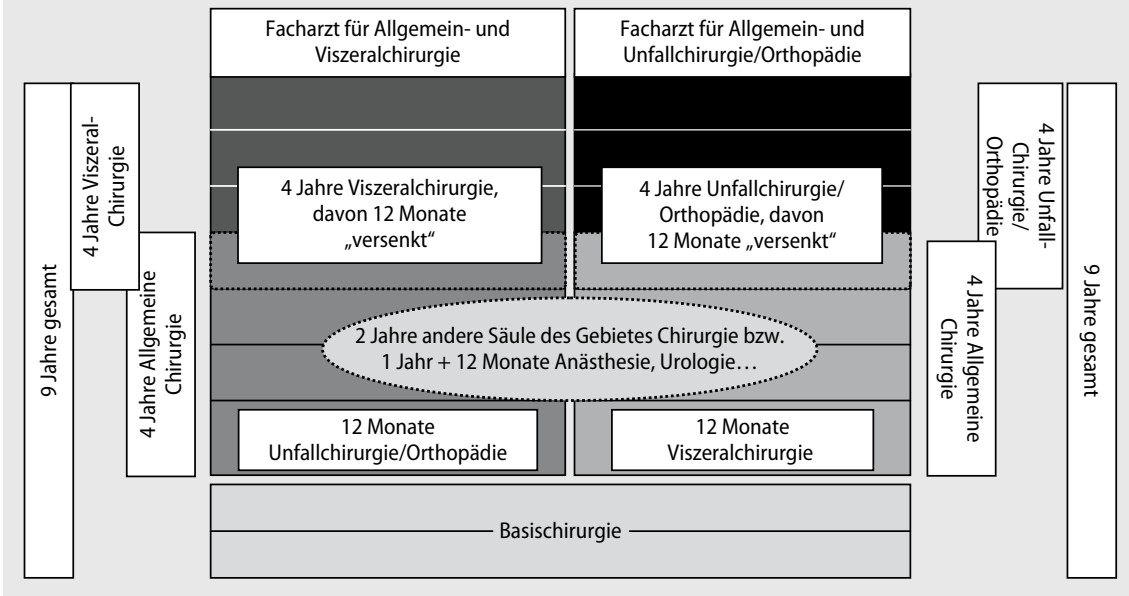

Abb. $3 \boldsymbol{\Delta}$ Lösungsweg doppelte Facharztqualifikation

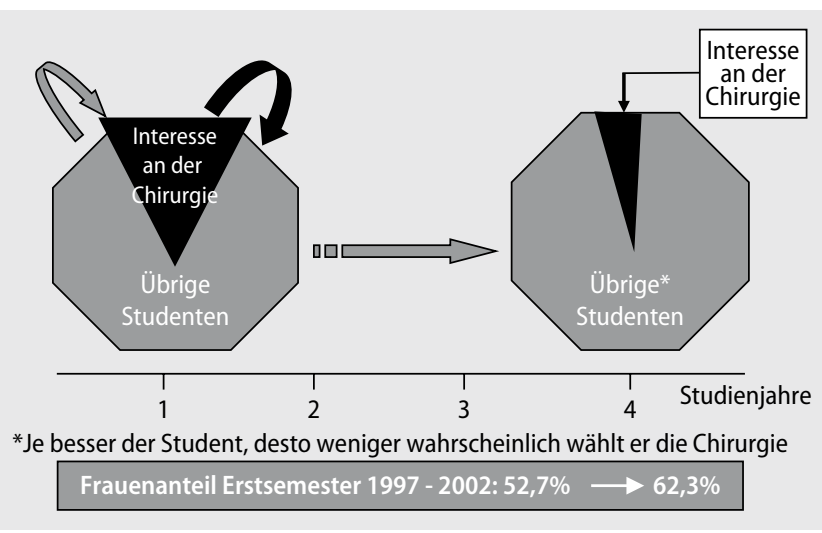

Abb. 4 Perspektiven hinsichtlich des chirurgischen Nachwuchses Frauenanteil Erstsemester 1997 - 2002: 52,7\% $\longrightarrow 62,3 \%$

Abb. 5 Paradigmenwechsel in den Versorgungsstrukturen

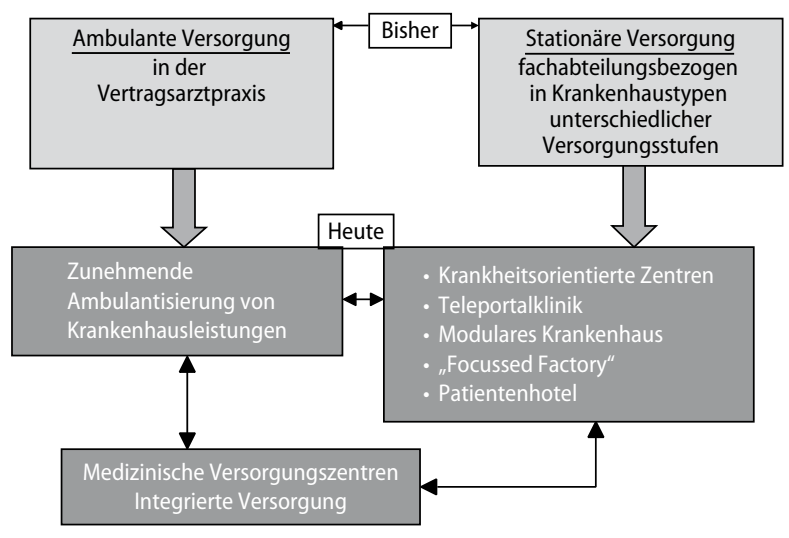

flut von Krankenkassen ausgefüllt. Für die eigentliche Aufgabe der Chirurgen, nämlich die Arbeit am Patienten auch außerhalb des Operationssaals und seine kompetente chirurgische Betreuung in der gesamten perioperativen Phase, bleibt immer weniger Zeit.

Hinzu kommt, dass es an gut strukturierten Weiterbildungsangeboten und qualifizierten Weiterbildern mangelt. Bei der Krankenhausfinanzierung über Fallpauschalen ist kein Ausgleich des höheren Aufwands für die Weiterbildung vorgesehen. Gute Weiterbildung aber braucht Anleitung und Zuwendung, sie kostet Zeit und damit Geld. Wenn Weiterbildungskosten nicht adäquat erstattet werden, werden die Zahl der Weiterbildungsstätten und die Qualität der Weiterbildung sinken, mit der Folge, dass sich wiederum 
Tab. 4 Chirurgische Weiterbildung: Handlungsbedarf: Steigerung von Qualität und Effizienz

\begin{tabular}{ll}
$\begin{array}{l}\text { Qualität } \\
\text { Qualifikationskriterien für Weiterbilder } \\
\text { und Weiterbildungsstätten mit Evaluation }\end{array}$ & $\begin{array}{l}\text { Effizienz } \\
\text { Abbau nichtärztlicher Tätigkeiten }\end{array}$ \\
$\begin{array}{l}\text { Weiterbildungscurriculum mit Planungs- } \\
\text { system für Weiterbildung (modular aufge- } \\
\text { bauter OP-Katalog, Logbuch) }\end{array}$ & $\begin{array}{l}\text { Straffung der notwendigen medizinischen Dokumen- } \\
\text { tationsarbeit }\end{array}$ \\
$\begin{array}{ll}\text { „Lernkurvenverlagerung vor den OP“ (Si- } \\
\text { mulatortraining, Skills Lab) }\end{array}$ & $\begin{array}{l}\text { Technische (EDV-) Unterstützung der Informationsbe- } \\
\text { schaffung und Dokumentation }\end{array}$ \\
$\begin{array}{l}\text { Kooperationsverträge zwischen Zentrum/ } \\
\text { anderen Krankenhäusern/Praxen }\end{array}$ & Verbesserung der Ablaufstrukturen \\
\hline $\begin{array}{l}\text { Gruppenermächtigungen } \\
\text { Prüfungen }\end{array}$ & $\begin{array}{l}\text { Anpassung der Weiterbildungsordnung (Mindestmen- } \\
\text { gen, Klinikstrukturen, Gebietsgrenzen) }\end{array}$ \\
\hline $\begin{array}{l}\text { Krankenhausentgelt: Weiterbildung nicht als zufälliges } \\
\text { Nebenprodukt der Arbeit }\end{array}$ \\
\hline $\begin{array}{l}\text { Weiterbildungsangebote als Wettbewerbsfaktor } \\
\text { Für materielle wie auch für geistige Investitionen gilt: Wer } \\
\text { nicht investiert, der verliert? }\end{array}$
\end{tabular}

weniger Ärzte zum Chirurgen ausbilden lassen. Um bei einem über 6o\%igem Frauenanteil unter den Studienabgängern auch mehr Frauen für die Chirurgie interessieren zu können, bedarf es dringend familienfreundlicherer Strukturen mit Teilzeitangeboten und Möglichkeiten der Kinderbetreuung. Weiterbildung darf nicht länger nur Nebenprodukt der Arbeit bleiben, sondern muss, wie dies gerade manche private Krankenhausträger vormachen, mit attraktiven Betreuungsangeboten zu einem Teil der Unternehmensstrategie werden $[1,2]$.

Viele Probleme sind aber auch „hausgemacht" und von den Chirurgen selbst zu verantworten. Nicht jeder gute Operateur ist auch ein guter Lehrer und nicht jede Einrichtung ist auch als Weiterbildungsstätte für den Erwerb der nötigen Facharztqualifikation geeignet. Eine verbesserte didaktische Qualifikation der Weiterbilder ist ebenso nötig wie eine Konzentration der Weiterbildungsangebote, die dann auch einen curricularen Ablauf mit entsprechenden Rotationen sicherstellen sollten. Mentorenkonzepte und Patenschaften sind v. a. für Chirurginnen und für Wiedereinsteiger wichtige Hilfen. Die chirurgisch-technische „Lernkurve“ sollte unter Nutzung moderner Simulatortechniken soweit als möglich vor den OP verlagert werden. An der Versorgungsrealität orientierte Curricula, mitarbeiterorientierte Zielvereinbarungen und eine Begleitdokumentation in den jetzt von den chirurgischen Fachgesellschaften eingeführten Logbüchern fördern die Qualität und die Transparenz der chirurgischen Weiterbildung (• Tab.4). Die Arbeit im Team innerhalb und außerhalb des Operationssaals muss bei immer komplexeren Operationstechniken und interdisziplinär abgestimmten Behandlungskonzepten gefördert werden. Dazu gehören auch eine verstärkte Zusammenarbeit und Arbeitsteilung zwischen Chirurgen und besonders qualifiziertem Assistenzpersonal.

\section{Delegation von Aufgaben und neue nichtärztliche Berufsbilder}

In Deutschland haben neben politischen Absichtserklärungen, wie etwa der Feststellung im Koalitionsvertrag, dass künftig nichtärztliche Heilberufe vermehrt in Versorgungskonzepte einbezogen werden sollten, v. a. die aktuellen Bestrebungen von Klinikträgern und Krankenhausleitungen, zunehmend ärztliche Leistungen von nichtärztlichem Personal erbringen zu lassen („Schwesternnarkose“), aber auch weitergehende Forderungen von Pflegeverbänden für erhebliche Unruhe gesorgt. Das aktuelle Gutachten des Sachverständigenrats wird sich ebenfalls intensiv mit einer stärkeren Aufgabenverteilung auf nichtärztliche Berufsgruppen und der medizinischen Versorgung durch multiprofessionelle Teams befassen. Von verschiedenen Anbietern liegen differente Ausbildungs- bzw. Weiterbildungsan- gebote für die neuen Berufsbilder vor, die sich auch über eine neu gegründete wissenschaftliche Gesellschaft verstärkt artikulieren wollen. Vor dem Hintergrund ungeklärter rechtlicher Rahmenbedingungen und Berufsanerkennungen, tradierter hierarchisch geprägter Rollenverteilungen, berufs- und standespolitischer Interessenvertretungen sowie einer vermehrt von ökonomischen Interessen geleiteten Aufgabenzuordnung an nichtärztliche Berufsgruppen wird so heftig über Grundsätzliches der Delegierbarkeit ärztlicher Aufgaben diskutiert, dass sinnvolle und längst nicht nur im Ausland bewährte Teamkonzepte auf der Strecke zu bleiben drohen.

Die Einbeziehung nichtärztlicher $\mathrm{Ge}$ sundheitsberufe in chirurgische Behandlungsteams mit unterschiedlichen Kompetenzleveln, wie sie z. B. in England für den so genannten Surgical Care Practitioner beschrieben sind, kann auch als Chance für einzelne chirurgische Fachgebiete gesehen werden. Dieses Assistenzpersonal, das immer nur arztunterstützend und nicht -ersetzend tätig werden kann, ist dem ärztlichen Dienst zuzuordnen, die ärztliche Supervision muss sichergestellt sein. Hier sind die einzelnen operativen Fachgesellschaften gefragt: Sie legen mit dem Ziel einer im interprofessionellen Team geleisteten Prozessverbesserung fest, welche Tätigkeiten in ihrem Fachgebiet sinnvoll und unter dem Aspekt von Sicherheit und Versorgungsqualität der Patienten delegiert werden können. Lehrpläne zur Vermittlung erforderlicher Qualifikationen müssen fachlich fundiert sein und sollten sich nicht primär an einer angestrebten Akademisierung auch in Form von Bachelor-Studiengängen orientieren.

Unbedingten Vorrang muss aber die seit langem nachdrücklich geforderte und immer noch unzureichend umgesetzte Entlastung der Ärzte von nichtärztlichen Tätigkeiten haben, die ihnen seit Jahren in zunehmendem Maß aufgebürdet wurden. Dazu gehören:

- Dokumentation und Kodierung zur Erlössicherung,

- Terminabstimmung,

- Sammeln von Befunden,

- Schreibarbeiten oder auch 
- Leistungen, die von Pflegekräften übernommen werden können wie Blutabnahmen, Verbände und die Anlage von Infusionen.

In Deutschland führen Ärzte Schwesternarbeiten und Schwestern Hilfsarbeiten aus. Dies ist vorrangig zu korrigieren. Arztassistenten stehen sonst im OP in Konkurrenz zu chirurgischen Assistenzärzten in der Weiterbildung, an denen die Bürokratie hängen bliebe. Weiterhin sind große Anstrengungen zur Verbesserung der chirurgischen Weiterbildung nötig, um einem sich abzeichnenden ärztlichen Personalmangel, auch eine der Hauptbegründungen bei der Forderung nach den neuen Assistenzberufen, entgegensteuern zu können [1].

\section{Chirurgie in neuen Versorgungsstrukturen}

Die Krankenhäuser stehen vor den größten Umstrukturierungsprozessen der letzten Jahrzehnte. Sie sind nicht nur Folge der gesetzlichen und ökonomischen Rahmenbedingungen (• Tab.5). Medizinischer Fortschritt mit zunehmender Spezialisierung, das Aufbrechen traditioneller Fachbereichsgrenzen, die Möglichkeit ambulanter Behandlung an Stelle klassischer stationärer Therapieformen, Verweildauerverkürzung, Fallzahlkonzentrationen unter Qualitäts- und Wirtschaftlichkeitsaspekten sowie verstärkte Markt- und Wettbewerbsmechanismen tragen wesentlich zu dieser Entwicklung bei.

Die Transformation vom traditionellen Krankenhaus von einer primär ärztlich geprägten und für eine gesicherte Breitenversorgung in seiner sozialen Bedeutung wenig hinterfragten öffentlichen Institution hin zum modernen, unter Wirtschaftlichkeitsaspekten zu führenden integrierten Gesundheitszentrum geht einher mit einem kontinuierlichen Kapazitätsabbau und einem an Intensität zunehmenden Verdrängungswettbewerb. Gleichzeitig kommt es zu einer enormen Leistungsverdichtung. Um sie bewältigen zu können, sind Investitionen in die Infrastruktur und Organisation erforderlich. Private Träger können hier rascher und flexibler reagieren. Bei einem prognosti-

\section{Tab. 5 Perspektiven: Entwicklung der Rahmenbedingungen}

\begin{tabular}{|c|c|}
\hline \multirow[t]{3}{*}{$\begin{array}{l}\text { Wissenschaftlicher } \\
\text { Fortschritt }\end{array}$} & $\begin{array}{l}\text { Komplexere Behandlungsverfahren zwingen zur Spezialisierung und } \\
\text { Aufgabenteilung interdisziplinär und interprofessionell }\end{array}$ \\
\hline & $\begin{array}{l}\text { Gewährleistung der hohen ärztlichen und medizinischen Qualitäts- } \\
\text { standards trotz ständig rückläufiger Ressourcen }\end{array}$ \\
\hline & $\begin{array}{l}\text { Entwicklung des medizinisch Machbaren und wirtschaftlich } \\
\text { Realisierbaren }\end{array}$ \\
\hline \multirow{4}{*}{$\begin{array}{l}\text { Druck der Krankenhaus- } \\
\text { finanzierung, ordnungs- } \\
\text { politischer Rahmen ab } \\
2009\end{array}$} & Liegezeitverkürzung (stringente Ablaufsteuerung, Arbeitsverdichtung) \\
\hline & Budget- und Leistungsvereinbarung \\
\hline & Wettbewerb um Patienten (Transparenz) \\
\hline & Qualitätsorientierte Vergütung \\
\hline \multirow{3}{*}{$\begin{array}{l}\text { Steigende Effizienzanfor- } \\
\text { derungen }\end{array}$} & Zentrenbildung, externe Kooperationen \\
\hline & Behandlungssteuerung \\
\hline & Bewertete Medizin \\
\hline \multirow{3}{*}{$\begin{array}{l}\text { Arztzahlentwicklung, per- } \\
\text { sonelle Engpässe, Arbeits- } \\
\text { zeitgesetz, Tarifabschlüsse }\end{array}$} & Fachübergreifende Dienste \\
\hline & Aufgabendelegation, neue Berufsbilder \\
\hline & 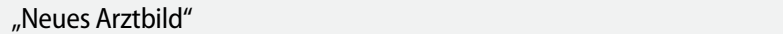 \\
\hline
\end{tabular}

Tab. 6 Medizinische Versorgungszentren (MVZ): Stand 01.01.2007. (Nach [5])

\begin{tabular}{|c|c|}
\hline \multirow{2}{*}{$\begin{array}{l}\text { Nahezu Verdopplung der Anzahl der MVZ } \\
\text { von Ende } 2005 \text { bis Ende } 2006 \\
\text { Allein im letzten Quartal } 2006 \text { Anstieg um } \\
\text { fast } 20 \% \text { (von } 562 \text { auf 666) }\end{array}$} & 2005: 341 \\
\hline & 2006: 666 \\
\hline $\begin{array}{l}\text { Verdopplung der Zahl der in MVZ tätigen } \\
\text { Ärzte auf }\end{array}$ & 2624 \\
\hline \multirow[t]{2}{*}{$\begin{array}{l}\text { Anwachsen der Anzahl der angestellten } \\
\text { Ärzte }\end{array}$} & $\begin{array}{l}\text { 2005: noch leichtes Übergewicht der Freiberufler } \\
\text { gegenüber angestellten Ärzten (496:474) }\end{array}$ \\
\hline & $\begin{array}{l}\text { 2006: wesentlich weniger Freiberufler als angestellte } \\
\text { Ärzte (928:1696) }\end{array}$ \\
\hline \multirow[t]{4}{*}{ MVZ-Gründung } & Vertragsärzte: $60 \% n$ \\
\hline & Krankenhäuser: 33\% \\
\hline & Krankenhäuser und Vertragsärzte: 3\% \\
\hline & Sonstige Träger: $4 \%$ \\
\hline Anzahl ärztlicher Mitarbeiter variiert & $2-16$ \\
\hline \multirow{3}{*}{$\begin{array}{l}\text { 1/4 der MVZ kooperiert mit nichtärztlichen } \\
\text { Leistungserbringern }\end{array}$} & Physiotherapeuten $11 \%$ \\
\hline & Sanitätshäuser 8\% \\
\hline & Ergotherapeuten 3\% \\
\hline
\end{tabular}

zierten Schwund von etwa 25\% der Krankenhäuser werden sie ihren Anteil weiter vergrößern. Mit Begriffen wie Teleportalklinik, Modulares Krankenhaus, „Concept Hospital“ oder „Focussed Factory“ werden klinische Organisationsstrukturen beschrieben, die nur noch wenig mit den bisherigen, nach Versorgungsstufen gegliederten Krankenhaustypen gemein haben (- Abb.5). Das moderne Krankenhaus wird zu einer Stätte intensiver, konzentrierter Medizin und dadurch einen jetzt noch vorhandenen Teil seiner Sozialfunktion, der über die Zeit der akuten Intervention zumindest teilweise hinausgeht, verlieren.

\section{Auswirkungen neuer Versorgungsformen}

Die gesetzlichen Vorgaben bewirken eine tief greifende Umverteilung von Krankenhausleistungen in Richtung „Ambulantisierung “ und Konzentration. Medizinische Versorgungszentren und integrierte Versorgungsmodelle ermöglichen ein Aufbrechen der bisher weitgehend abgeschotteten Versorgungssektoren mit ihren negativen Auswirkungen nicht nur auf die Effizienz der Leistungserbringung, sondern auf eine auch aus Patientensicht sinnvolle engere Kooperation der unterschiedlichen Leistungsbereiche. Eine bisher nur in Ansätzen bestehende integrierte Versorgung z. B. durch regionale 


\section{Neuer Facharzt}

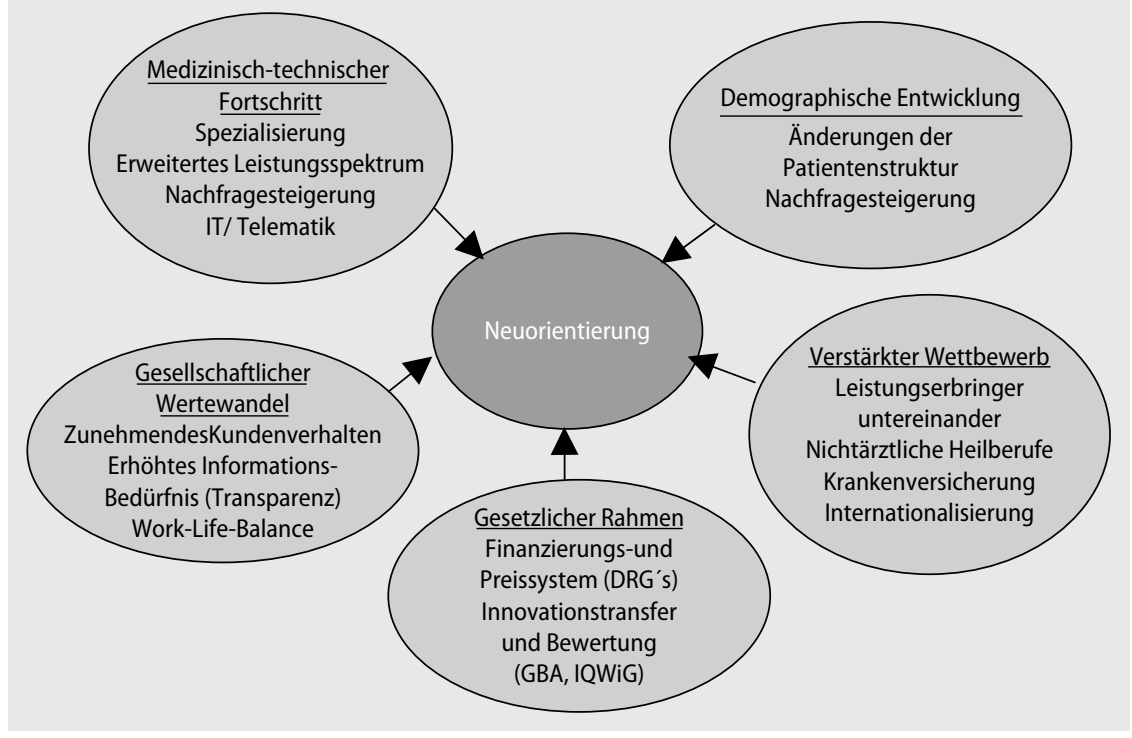

Abb. $6 \Delta$ Chirurgie in neuen Strukturen: professionelle Neuorientierung

Arztnetze (horizontale Integration) wird durch eine Kooperation zwischen Krankenhäusern und niedergelassenen Ärzten sowie mit Rehabilitationseinrichtungen (vertikale Integration) eine deutliche Ausweitung erfahren. Für die Medizinischen Versorgungszentren ist dies bereits Realität ( $\bullet$ Tab. 6). Insbesondere für Chirurgen und Orthopäden eröffnen sich hier interessante ambulante Tätigkeitsfelder ohne die investiven Belastungen, wie sie für eine eigenen Praxis eingegangen werden müssen.

\section{Spezialisierung, Fallzahlkonzent- ration und Zentrumsbildung}

Der medizinische Fortschritt zwingt zur Spezialisierung und Zentrenbildung. Nur so lässt sich eine Versorgungsqualität nach aktuellem wissenschaftlichem Stand für die Patienten sicherstellen. Kompetenzzentren müssen dabei nicht ausschließlich auf Universitätskliniken oder Häuser der Maximalversorgung beschränkt sein.

Als umstrittenes Steuerungselement gilt die Mindestmengenvereinbarung. Bisher fallen darunter nur Eingriffe mit hohem Risikoprofil, für die ein „high volume"-Effekt belegt ist. Eine Ausweitung auf andere Indikationsbereiche ist umstritten, ausreichende Studien zur Auswirkung von Mindestmengenvorgaben auf die flächendeckende Versorgung fehlen. Die DRG-Einführung wird den ökonomischen Druck verstärken. In letzter Konsequenz wird das Vergütungssystem zunehmend den Standort sowie die Art und den Umfang der stationären Leistungserbringung bestimmen. Weiterhin wird die zunehmende Transparenz durch die pflichtgemäß zu veröffentlichenden Qualitätsberichte die Auswahlmöglichkeit für Patienten und Kostenträger verbessern und v. a. auf der Basis von Mengenvergleichen zu Rankings unterschiedlichster Art genutzt werden und so Markt und Wettbewerb stärken.

Spezialisierung ist ein unaufhaltsamer Trend. Das Krankenhaus und auch integrierte Versorgungseinrichtungen sind dabei auf dem Weg zur Ein-Organ-Spezialisierung bzw. zu krankheits- oder methodenspezifischen Zentrenbildungen. Im Unterschied zur gewohnten Abteilungsorganisation, die sich mit ihren Fachbereichen weitgehend an ärztliche Professionen anlehnt, sind Zentren Wirklichkeit gewordene Antworten gleichermaßen auf bestehende medizinische und betriebswirtschaftliche Herausforderungen. „Echte“ Zentren zeichnen sich dadurch aus, dass sie die medizinische Qualität, z. B. durch die Zusammenarbeit verschiedener Disziplinen innerhalb des Zentrums, steigern oder durch Bündelung von Ressourcen Einsparungen erzielen. Um sich optimal zu positionieren, müssen Krankenhäuser nach außen klare Strukturen schaffen und nach innen die interdisziplinäre Zusammenarbeit optimieren. Sie müssen dabei innere Grenzen zwischen den Einrichtungen und Abteilungen innerhalb des Hauses überwinden. Die bis- herige Abteilungsstruktur taugt nicht für die Zukunft. Sie ist unter dem Aspekt der neuen Herausforderungen zu wenig effizient und patientenorientiert [5].

\section{Professionelle Neuorientierung: welche Chirurgen für welche Aufgaben?}

Die künftige Facharztweiterbildung und die sich verändernden Krankenhausstrukturen stehen in einer engen Beziehung. Die Vermittlung der gesamten Weiterbildungsinhalte an einer Fachabteilung unter Leitung eines dazu befugten Weiterbilders wird als Regelfall der Vergangenheit angehören. Im Rahmen von Verbundermächtigungen müssen effiziente Rotationssysteme bei einer klaren curricularen Gliederung der Weiterbildung an die geänderten Abteilungs- und Versorgungsstrukturen angepasst werden. Die Frage der Zukunft heißt: Für welche Versorgungsaufgaben müssen wir wie qualifizierte Chirurgen weiterbilden? Die längerfristige Tragfähigkeit des derzeitig gültigen Säulenmodells der Weiterbildungsordnung erscheint ebenso fraglich wie die Sicherstellung einer flächendeckenden Grund- und Notfallversorgung.

Die Konkurrenz zwischen medizinischen und kaufmännischen Denkansätzen wird sich massiv verschärfen. Alle nicht zwingend notwendigen Leistungen eines Krankenhauses werden unter Effizienzgesichtspunkten überprüft werden. Der Druck, Versorgungsstrukturen zu ändern und Leistungsangebote zu konzentrieren, ist die spürbare Auswirkung dieser Ökonomisierung. Darüber hinaus erfordern weitere Einflussfaktoren auf der gesellschaftlichen und politischen Ebene eine professionelle Neuorientierung (- Abb. 6).Sich rechtzeitig darauf einzustellen, heißt nicht nur Risiken zu begegnen, sondern auch Chancen zu nutzen.

\section{Fazit}

Die Ära der Chirurgen, die nur für die Chirurgie gelebt haben, ist endgültig vorbei. Das Berufsbild darf vor dem Hintergrund der sich rasch wandelnden gesellschaftlichen Veränderungen nicht stehen bleiben. Künftige Generationen spezialisierter Chirurgen werden 
in selbstbewusster Wahrnehmung ihrer Spezialkompetenz ihre eigenen Grenzen erkennen und die enge Kooperation mit anderen Spezialisten in krankheitsorientierten Zentren als selbstverständlichen Teil ihres Berufsbildes sehen. All dies muss von den Verantwortlichen auch vorgelebt und vermittelt werden.

\section{Korrespondenzadresse}

Prof. Dr. H. Bauer

Deutsche Gesellschaft für Chirurgie, Luisenstraße 58/59, 10117 Berlin

H.Bauer@dgch.de

Interessenkonflikt. Der korrespondierende Autor gibt an, dass kein Interessenkonflikt besteht.

\section{Literatur}

1. Bauer H (2004) Die derzeitige Facharztweiterbildung und die zukünftige Krankenhausstruktur. In: Wolff H (Hrsg) Die zukünftige Chirurgie zwischen Spezialisierung und High-Volume-Hospitals. Kaden, Heidelberg, S68-75

2. Bauer $H$ (2006) Defizite in der chirurgischen Weiter- und Fortbildung:Weiterbildungsleiter/Weiterbildungseinrichtung. In: Wolff $\mathrm{H}$ (Hrsg) Defizite in der chirurgischen Weiter- und Fortbildung. Wissen und Erfahrung. Kaden, Heidelberg, S 63-68

3. Bauer $\mathrm{H}$ (2007) Was wird aus der Allgemeinen Chirurgie? Mitt Dtsch Ges Chir 36: 137-140

4. Bauer $\mathrm{H}$ (2007) Versorgungsengpässe in der Chirurgie. Kommentar. Chirurg 78: 259-260

5. Pongs K, Jüttner C, Baierlein J et al. (2007) Die alte Abteilungsstruktur taugt nicht für die Zukunft. Zentrenbildung ist die Basis für Leistungsprofilierung und Qualitätssteigerung. F\&W 24: 278-282 